\title{
Project Quality Management Development and Process Approach Theory Research Overview
}

\author{
Qingchen Zhang*, Yunfeng Lu
}

Space Engineering University, Beijing, China

*Corresponding author.Email: ccbtv@foxmail.com

\begin{abstract}
To help managers enhance their understanding and attention to quality, this paper summarized the development and research status of quality management theory. Through summarizing the research achievements of scholars in the field of quality management, exploring the history of quality management theory's development, and understand its application in industrial, construction engineering, scientific research, business administration and education practice, analysed the application situation of process approach in quality management system. It is found that modern quality management theory has been highly recognized and widely used in all aspects of human society, especially in the field of engineering projects.
\end{abstract}

Keywords: Quality Management, Process Approach, Project Management, Research Overview.

\section{项目质量管理发展与过程方法理论研究综述 \\ 张清晨 ${ }^{*}$, 陆云峰}

航天工程大学, 北京, 中国

*通讯作者. 邮箱: ccbtv@foxmail.com

\section{中文摘要}

论文旨在梳理归纳质量管理理论的发展和研究现状, 帮助管理者增强对质量的理解和重视。通过总结各国学者 们在质量管理领域的研究成果, 探寻质量管理理论的发展历史, 了解其在工业生产、工程建设、科学研究、企 业发展以及教育教学领域的应用实践情况, 重点针对质量管理体系中的过程方法的应用现状进行了分析。研究 发现，现代质量管理理论伴随实践活动，在人类社会的各个方面均得到了高度认可和广泛应用，尤其在工程项 目领域取得了显著的效果。

关键词: 质量管理, 过程方法, 项目管理, 研究综述

\section{1. 现代项目质量管理的发展}

\section{1. 质量管理理论的发展进程}

通常来说, 质量管理的发展经历了三个阶段, 即: 质量检验阶段、统计质量管理阶段和全面质量管理阶 段。上个世纪 20 年代初期, 主要是通过检验剔除不
合格品达到保证产品质量的目的，此时的质量管理处 于初级阶段。不久, 泰勒提出科学管理理论, 同时建 立了专职质量检验制度，同时期的休哈特博士提出统 计过程控制理论，并首创“控制图”进行过程监控，为 质量控制理论奠定了基础, 道奇提出的抽样检验理论 构成了质量检验理论的重要内容。从休哈特发明质量 控制图直到 20 世纪 50 年代末, 是统计质量管理阶段, 
强调“用数据说话”和应用统计方法进行质量管理。上 个世纪中叶, 伴随科学技术的飞速发展, “全面质量管 理”理论诞生并不断发展完善。全面质量管理强调全 面、全过程和全员参加。

目前, 国际标准化组织 (ISO) 在 ISO 9001:2015 中 对质量管理的定义为: 关于质量的管理。通常包括: 制定质量方针和质量目标, 以及通过质量策划、质量 保证、质量控制和质量改进实现这些质量目标的过程。 这就是指挥和控制组织活动的基本内容 ${ }^{[1]}$ 。

世界各国的质量管理大师们对于质量管理有着不 同的理解。朱兰 (Joseph M. Juran) 博士认为质量管理 活动中应用着质量计划、质量控制、质量改进三个管 理过程, 又称之为“质量管理三部曲”; 戴明 (W. Edwards Deming）博士提出了质量管理理念的 14 条 要点; 阿曼德·费根堡姆 (Armand Vallin Feigenbaum) 认为, 质量意味着客户使用和售价最佳, 并提出了质 量管理四个基本原则; “零缺陷之父”克劳士比 (Philip B. Crosby) 认为绝对没有理由在任何产品和服务中存 在错误和缺陷, 即“零缺陷”; 休哈特 (W. A. Shewhart) 结合统计学、工程学、经济学, 开创了统计质量控制 的新领域, 其“计划一执行一检查一行动循环”的观点 被戴明和其他人广泛采用; 石川馨(Ishikawa Kaoru)提 出“品质控制 (QC) 小组”的概念, 并推动帕累托图、 因果图等工具的使用以优化质量控制。质量管理大师 们的质量观点为质量管理提供了基本原则 ${ }^{[2]}$ 。各种质 量观点中许多思想是共通的, 虽然存在一些差异, 但 是质量先驱对质量追求方向是一致的, 都提倡对质量 永无终止的改进, 为现在的质量理论做出了巨大的贡 献 ${ }^{[2]}$ 。

对于现代项目管理的实践活动而言, 项目质量不 仅包括项目的成果，也包括项目管理的质量 ${ }^{[3]}$ 。美国 项目管理协会（PMI）《PMBOK 指南》（第六版）认 为项目质量管理包括把组织的质量政策应用于规划、 管理、控制项目和产品质量要求, 以满足相关方目标 的各个过程 (包括规划质量管理、管理质量、控制质 量三个过程）。此外, 项目质量管理以执行组织的名 义支持过程的持续改进活动。

\section{2. 质量管理在中国的发展进程}

在中国, 质量管理的维形则可以追溯到上千年前, 在古代典籍中记载有大量有关产品质量的规定、要求 等, 这是质量管理的初期体现。自新中国成立至 1977 年, 我国的质量管理只是处于质量检验阶段, 直到 1978 年前后企业生产质量还存在诸多问题; 1978 年 11 月, 我国经济代表团在考察日本企业管理情况后深 刻认识到“全面质量管理”在提高质量、降低成本、提 升效率方面的重要作用; 1979-1989 年, 我国开始引 进并推广全面质量管理, 并于 1979 年颁布了《优质 产品奖励条例》；1989-1999 年，《中华人民共和国产 品质量法》《质量振兴纲要》《国务院关于进一步加 强产品质量工作若干问题的决定》先后颁布, 标志着
我国进入全面质量管理的普及和深化阶段; 1999 年至 今, ISO9000 族标准引入国内, 国家先后颁布 GB/T19000 族标准、《卓越绩效评价准则》《卓越绩 效评价准则实施指南》, 这些推动了我国质量管理工 作的开展, 标志着我国全面质量管理进入发展和创新 阶段 ${ }^{[4]}$ 。

\section{2. 质量管理研究现状}

\section{1. 国外质量管理研究现状}

国外学者对于质量管理由广泛且深刻的研究。 Lassaaad Lakhal 等对 24 个项目中的领导作用、质量组 织、员工培训、员工参与、顾客关注、信息和分析以 及质量体系改进等 7 个方面进行了分析, 发现领导作 用对项目质量管理起到了关键的影响 ${ }^{[9]}$; Hale Kaynak 验证了全面质量管理实践中各要素之间的直接和间 接关系, 以及这些实践对企业绩效的影响, 他认为领 导承诺的缺失可能导致组织全面质量管理系统的失 效 ${ }^{[10]}$; Rui Sousa 等认为有必要对质量管理实施内容 进行更多的实证研究, 从针对特定项目的研究、以理 论支撑进而获得丰富的知识、反思审查三个方面入手 [11]; Fred Appiah Fening 等针对加纳 116 家中小企业 的 5 项绩效指标分别调查了 7 项质量管理实践。得出 “质量管理的所有变量均与绩效呈显著正相关关系” 的结论, 并且证实了质量管理是由各种变量组成的, 这些变量结合在一起形成了质量管理, 实践必须以循 序渐进的步伐进行 ${ }^{[12]}$ 。

总的来说, 其他国家对于项目管理和项目质量管 理的研究起步较早, 研究也更为深入, 形成了系统的 知识体系并且应用广泛, 对世界项目管理的发展有着 深刻影响。与此同时, 学者们也在各领域的研究中对 质量管理理论进行不断的发展和完善, 尤其是基于大 量样本的数据分析, 对传统质量管理理论有了更为科 学的评判, 这些都推动了世界项目管理学科与质量管 理理论的发展, 同时对国内的研究产生了深刻影响。

\section{2. 中国质量管理研究现状}

在项目质量管理领域, 中国的学者有诸多的研究 实践。学者周娅对项目质量管理和一般质量管理两者 间的联系和区别进行了研究。她建议应对项目质量管 理与一般质量管理相互关系有正确认识, 在项目质量 管理中灵活引入一般质量管理的理论和方法, 进而促 进项目目标顺利实现 ${ }^{[5]}$ 。吕卫胜提出, 工程项目质量 管理对工程项目各个阶段所使用整套质量管理体系 和工作方法进行管理, 进而保证预期工程目标和提高 整体工程质量 ${ }^{[6]}$ 。殷小惠认为, 为项目的用户和其他 项目相关者提供高质量的工程与服务, 实现项目目标、 使用户满意是项目质量管理的目的 ${ }^{[7]}$ 。张子海认为, 保证项目质量目标的实现是项目质量管理的目的 ${ }^{[8]}$, 他提出：预防和事先控制、过程检验对于防止质量问 题更加有效, 而避免质量问题的费用通常比纠正它们 低得多 ${ }^{[8]}$ 。 
总的来说，虽然中国自古以来都有对于管理活动 与质量管理的实践, 但现代项目管理的引入和运用起 步较晚, 甚至直到 20 世纪 60 年代之前一直未能形成 科学的理论。中国的项目质量管理理论受到了他国学 者研究结果的极大影响, 尤其是改革开放以来, 中国 的学者们纷纷将项目质量管理理论运用于项目实践 当中, 取得了较好的成果, 推动了中国项目管理和质 量管理领域的发展。

\section{3. 过程方法理论}

过程是一组将输入转化为输出的相互关联或相互 作用的活动, 并在将输入转化为输出的过程中产生价
值。任何利用资源并通过管理, 将输入转化为输出的 活动均可视为过程 ${ }^{[13]}$ 。按照这样的方式, 将活动作为 相互关联、功能连贯的过程组成的体系来理解和管理, 可更加有效和高效地得到一致的、可知的结果 ${ }^{[14]}$ 。单 一过程的各要素及其相互作用如图 1 所示, 每一过程 均有特定的监视和测量检查点以用于控制[14]。前序过 程的输出结果作为当前活动的输入内容, 经过当前活 动产生新的输出结果, 而新的输出结果交予下一个过 程, 又成为后续过程活动的输入内容。可以看到的是, 在单一过程中, 每个阶段都设置了可能用于监视和测 量绩效的控制和检查点, 以使得每个环节中都能对过 程执行质量进行有效控制。

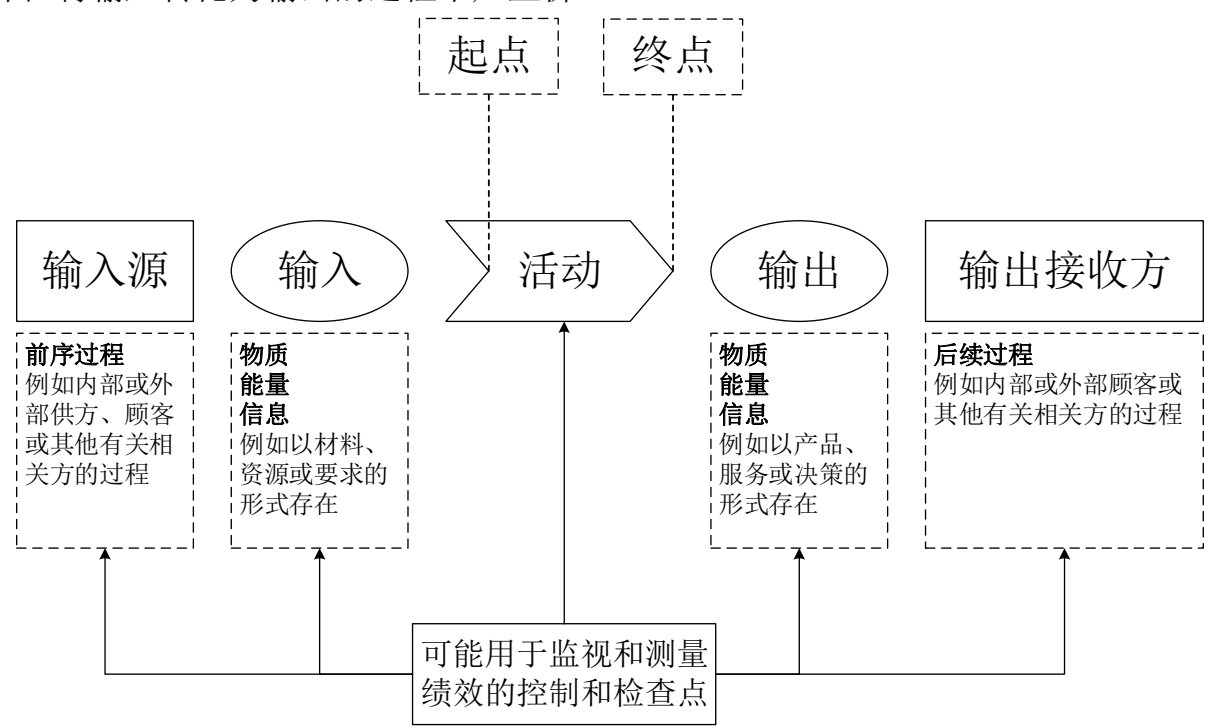

图 1 单一过程要素示意图

国外学者 Dhanasekharan Natarajan 教授在专著 《ISO 9001 Quality Management Systems》中以单一过 程为例, 对其控制方式进行了解读。他提出, 需要对 于过程产物进行检查, 对未达验收标准的产物进行分 析测量, 同时寻找过程方面的问题, 对其进行纠正或
改进, 通过调整输入内容、控制方式与过程, 以使得 该项过程的输出满足要求。Dhanasekharan Natarajan 教 授在对于单一过程要素的控制中运用了反馈调节的 思路, 并强调了对于过程产物以及过程本身的测量分 析和改进，这也是对于过程方法整体运用的基本思路。

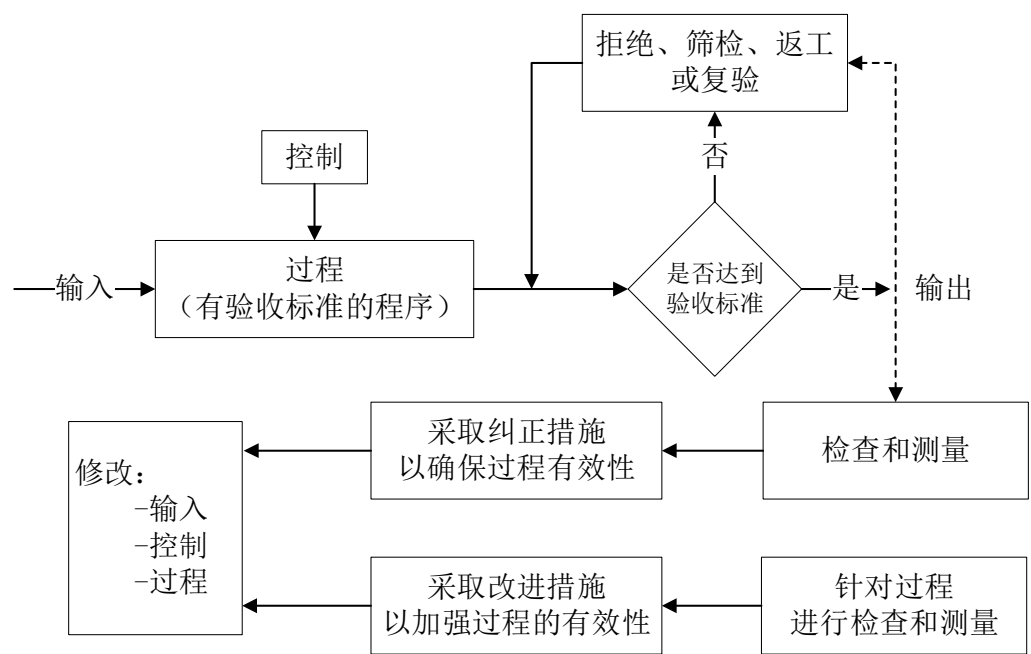

图 2 Dhanasekharan Natarajan 教授对过程的解读

过程方法是 GB/T19000 族标准所阐述的质量管理 七项原则之一，同时也是《GB/T19001-2016 质量管
理体系要求》中所采用的方法。理解过程和过程方法 的概念，是科学运用的前提。过程方法是将项目的所 
有工作视为若干个“输入一处理一输出”的过程, 将相 互关联的过程作为一个体系加以理解和管理, 将客户 的要求和各类法律规范标准作为输入项作为对产品 （可交付物）的限制条件, 经过项目活动生成的产品 （可交付物）作为成果交付给客户并达到客户满意的 要求, 整个“输入一处理一输出”的过程即为产生价值 的增值活动。
采用过程方法使组织能够控制过程之间相互关联 和相互依赖的关系, 以提高组织绩效。对于一组活动 而言，过程可分为主要过程和支持性过程，抓住对主 要过程的控制是把握整体过程的关键所在。经典的过 程方法模式如图 3 所示。

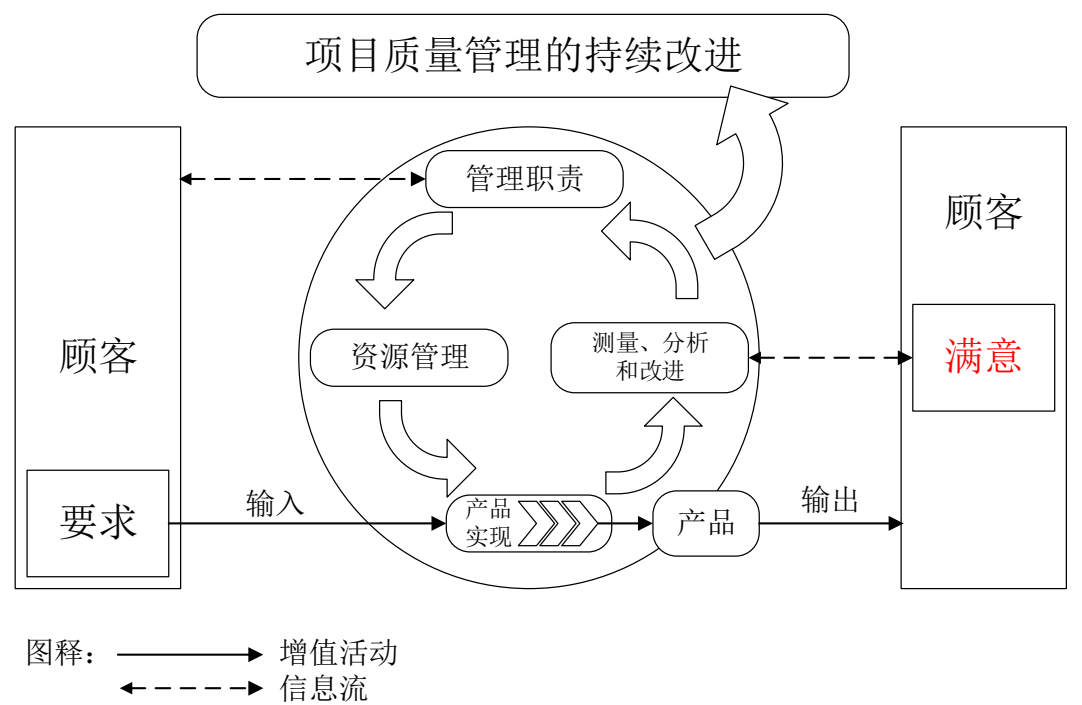

图 3 经典的过程方法模式

传统的质量管理偏向于对关键要素进行控制, 由 于各个质量要素是孤立的, 缺乏联系, 因此, 在实施 质量控制时缺乏连贯性, 不能做到瞻前顾后, 对于项 目整体质量的管理缺乏全局性的掌控; 而采取针对过 程的质量控制方法, 则在针对要素控制的基础上, 将 分散的质量控制要素融入到过程中, 并通过一系列活 动将它们串联起来、形成连点成线、铺线成面的局面, 有效保证了质量管理的整体性和连续性, 同时, 在过 程方法中将管理活动构建成动态、闭环控制的模式, 让项目运转可控, 并且有反馈、有改进, 在动态循环 中不断提高质量。对于质量管理而言, 管理更加高效、 控制更加精准, 同时, 对于质量问题的改进也更有针 对性和科学性。

\section{4. 过程方法理论研究现状}

经典的过程方法要求在产品 (可交付物) 的形成过 程中, 将所有的活动划分为管理职责、资源管理、产 品实现、测量分析与改进等四个阶段, 四个阶段的活 动相互衔接、形成闭环, 并在循环中不断改进。在循 环过程中，需注意到客户与项目组之间的信息流交换， 表现为项目组与客户的密切沟通, 及时了解客户需求 并寻求反馈, 不断纠偏改良, 使得最终成果得以满足 客户要求。同时, 在整个闭环的优化过程中, 亦使得 项目组的质量管理能力不断改进提升。

最新版 ISO9001:2015 对过程方法模式进行了进一 步优化, 新版过程方法中更加强调领导作用, 同时也 更加关注过程导向、基于风险的思维和顾客导向。
过程方法已经在产品制造、建筑工程、航天项目等 领域有了广泛运用。同时, 国内学者对过程方法的应 用范围、运用优点和使用时的注意事项都做了详尽的 分析和阐述:

白世贞、陈锦、郭同生等学者对于过程方法的运用 价值做出了总结, 并表达了自己的观点。白世贞认为, 过程方法是企业科学管理方法的核心，具有普通的适 用意义 ${ }^{[15]}$; 陈锦认为, 过程方法最大的优点就是: 对 系统中单个过程之间的联系以及过程的组合和相互 作用进行连续的控制 ${ }^{[16]}$; 郭同生认为, 使用“过程方 法”有利于建立联系、增进沟通、提高效率、便于改进 等 $[17]$ 。

李惠芳等学者对于过程方法运用当中需要注意的 问题进行了梳理和总结。她认为, 在使用过程方法时, 需要按照过程方法的要求系统地识别过程, 并且明确 过程的顺序、接口、关系及相互作用 ${ }^{[18]}$; 范军旗提出, 在考虑应用过程方法时, 必须要抓住影响过程的主要 因素、要注意以往类似过程的相关信息、要加强对特 殊过程的控制[19]。杨辉、金华彰等则认为, 过程方法 在有效控制质量管理体系中应明确过程方法实施的 范围和要求、要实施过程控制的连续性、要体现过程 管理的系统性、抓好过程管理的程序化和定量化、要 注重体系运行的有效性、采用过程方法开展质量审核 [20-21]。

当前，过程方法在项目管理中的应用还存在一些 问题, 有学者对问题现状进行了分析并提出了解决思 路。针对“项目参与者对过程方法的理解不够、对过程 
方法的基本概念模糊、受传统方法约束等，使得过程 方法实际应用效果不佳”的现状, 学者张小海通过比 较分析过程与活动、过程与职能部门、过程与过程方 法、过程与过程系统、组织固有过程与过程思路等之 间的关系，提出了过程方法在应用时应关注的问题， 为过程方法的实践活动提供了指南 ${ }^{[22]}$ 。

\section{5. 结语}

伴随着实践活动的不断深入, 质量管理理论历经 近百年的发展已经愈发成熟, 并且向着更先进的方向 不断改进，尤其是在现代项目管理领域，过程方法等 先进质量管理思想的融入, 有效提高了项目的质量和 完成效率，促进了项目管理科学的发展。

\section{REFERENCES}

[1] Zhang Yong, Chai Bangheng. ISO 9000 quality management system 3rd edition [M].Beijing: China Machine Press, 2016.

[2] Lin Youhuai. Summary of Quality Management of Information Network Engineering Project Construction $[\mathrm{M}]$. Beijing: Posts and Telecommunications Press, 2011.

[3] Bai Sijun. Introduction to Modern Project Management in Textbooks of Project Management Series in Colleges and Universities [M].Beijing: Publishing House of Electronics Industry, 2013.

[4] SUN Lei. Practical Complete Book of Quality Management $[\mathrm{M}]$. Beijing: Posts and Telecommunications Press, 2011.

[5] Zhou Ya, Huang Shaolin. Comparative Study on Project Quality Management and General Quality Management [Z]: Market Modernization, 2009.

[6] Lv Weisheng. Research on Quality Management of Power Transmission Line Project in Heze Region [D].North China Electric Power University, 2014.

[7] Yin Xiaohui. Research on the Key Influencing Factors of Construction Project Quality Management [D].Shandong University, 2012.

[8] Zhang Zihai. Analysis of project quality management [J]. Project Management Technology, 2009, 7(8): 88-92.

[9] Lassa^ad Lakhal; Federico Pasin; Mohamed Limam. Quality management practices and their impact on performance[J]. International Journal of Quality \&Reliability Management, 2006, 23(6): 625-646.

[10] Hale Kaynak.The relationship between total quality management practices and their effects on firm performance[J].Quality Control and Applied Statistics.2004,Vol.49(NO.2): 175-176.
[11] Sousa, R.*; Voss, C.A.. Quality management revisited: a reflective review and agenda for future research[J]. Journal of Operations Management.2002, Vol.20(No.1): 91-109.

[12] Fred Appiah Fening; Gordana Pesakovic; Pesi Amaria. Relationship between quality management practices and the performance of small and medium. size enterprises (SMEs) in Ghana[J].International Journal of Quality and Reliability Management.2008,Vol.25(No.6-7)

[13] Guidance on Application of Quality Management System GB/T19001 -- 2016. [S].

[14] General Administration of Quality Supervision, Inspection and Quarantine of the People's Republic of China, Standardization Administration of China.Quality management system requirements. [S], 2016.

[15] Bai S Z. On the process method of quality management [J]. Business Research, 2002(12): 1618.

[16] CHEN J, WANG Z Q. Application of process method in scientific research projects [C]// Proceedings of the Second Youth Science and Technology Forum of China Hydraulic Society: Yellow River Water Conservancy Press, 2005: 628632.

[17] Guo Tongsheng. Application of "Process Method" in Quality Management System [J].World Standardization and Quality Management, 2005(5): 15-19.

[18] Li Huifang. Application Analysis of Process and Process Method in Quality Management System [J].Manufacturing Informatization in China, 2012(17).

[19] Fan Junqi. On the Application of Process Method in Quality Management System [J].Value Engineering, 2015, 34(10): 298-299.

[20] Jin Huazhang. Establishing, Implementing, Maintaining and Improving Quality Management System Using "Process Method" [J].Electronic Quality, 2005(4): 52-55.

[21] YANG H. Application of process method in quality management system [J]. Information Technology and Standardization, 2006(9): 57-60.

[22] Zhang Xiaohai, Xue Kui. Process and Process Method in Quality Management System [J].Business Economics, 2012, 31(10): 76-78. 